\title{
On the Foundational Assumptions of Modern Physics
}

\author{
Benjamin F. Dribus \\ Louisiana State University \\ (email: bdribus@math.lsu.edu)
}

August 12, 2012

\begin{abstract}
General relativity and the standard model of particle physics remain the most fundamental physical theories enjoying robust experimental confirmation. The foundational assumptions of physics changed rapidly during the early development of these theories, but the challenges of their refinement and the exploitation of their explanatory power turned attention away from foundational issues. Deep problems and anomalous observations remain unaddressed. New theories such as string theory attempt to resolve these issues, but are presently untested. In this essay, I evaluate the foundational assumptions of modern physics and propose new physical principles. I reject the manifold structure of spacetime, the existence of an independent time parameter and static background structure, the symmetry interpretation of covariance, the commutativity of spacetime, and a number of related assumptions. The central new principle I propose is called the causal metric hypothesis. The classical version of this hypothesis states that the metric properties of spacetime, up to overall scale, arise from the binary relation generating the causal order. The quantum version states that the phases associated with congruence classes of directed paths in causal configuration space are determined by the causal relations of their constituent universes.
\end{abstract}

\section{Introduction}

Relativity and quantum theory emerged from mathematical seeds in the works of Gauss, Riemann, Cayley, Hilbert, and others, were incorporated as physical theories by Einstein, Bohr, Heisenberg, Schrödinger, and their contemporaries, and matured as definitive predictive systems in the form of modern general relativity and the standard model of particle physics in the second half of the twentieth century. Among theories enjoying robust experimental confirmation, these two theories represent our deepest understanding of fundamental physics. The rapid alteration of foundational assumptions characterizing the early development of these 
theories later diminished while their fruit was harvested. The complete unification of relativity and quantum theory was gradually understood to be a particularly intractable problem after numerous optimistic early attempts, and an abundance of new experimental results amenable to description within the existing framework of quantum field theory decreased motivation for radical new departures.

Recently the triumphs of quantum field theory have slowed, and unexplained phenomena such as dark matter and dark energy hint at new physics. In this environment, long-acknowledged foundational problems have gained new urgency. The microscopic structure of space, the nature of time and its relation to causality, the quantum theory of gravity, and the unified description of the forces of nature, have all attracted increased scrutiny. Untested new theories seek to address these issues, often by means of new assumptions as alien to established physics as the assumptions of relativity and quantum theory are to the Newtonian paradigm. Among these new theories, string theory [1] abolishes point particles and introduces new dimensions, symmetries, and dualities; loop quantum gravity [2] proposes the quantization of classical spacetime; noncommutative geometry [3, 4] interprets spacetime as a noncommutative space; entropic gravity [5] attributes gravitation to the second law of thermodynamics; and causal set theory [6, 7] discards classical spacetime in favor of partially ordered sets. While limited, this list represents many of the general approaches under active investigation.

In this essay I evaluate the foundational assumptions of modern physics and offer some speculative new principles, partially overlapping some of the theories mentioned above. Among the assumptions I reject are the manifold structure of spacetime, the evolution of systems with respect to an independent time parameter, the existence of a static background structure, the symmetry interpretation of covariance, the transitivity of the binary relation generating the causal order 11 and the commutativity of spacetime. The central new principle I propose is the causal metric hypothesis, which has both a classical and a quantum-theoretic version. The classical version states that the metric properties of spacetime, up to overall scale, arise from the binary relation generating the causal order ${ }^{2}$ The quantum version applies to the sum-over-histories method originated by Feynman, and states that the phases associated with congruence classes of directed paths in causal configuration space are determined by the causal relations of their constituent universes.

This essay is organized as follows: in section 2 I identify and discuss the foundational assumptions of modern physics, including six general principles and a number of specific assumptions of particular modern theories. In section 3, I review some empirical phenomena, including dark matter, dark energy, and matter-antimatter asymmetry, which are unaccounted for by established theories. In section 4, I reject several existing assumptions, with motivation provided by the previous two sections. In section 5, I propose new physical principles. The fundamental structure of spacetime is the central focus of both the rejected assumptions and the new principles. In section 6, I remark on the empirical status of these assumptions and principles in light of the current state of experimental physics, and suggest how the ideas presented here might find their way into the laboratory. 


\section{Identifying the Foundational Assumptions}

In the universe of scientific thought, ideas from mathematics, philosophy, and the empirical realm combine in the form of general physical principles, which crystallize into the formal postulates of physical theories, while remaining colored and sometimes distorted by the interpretations and prejudices of their intellectual environment. These principles, postulates, and ancilla are all foundational assumptions in the sense that scientific progress depends critically, and to some degree independently, on each, yet they also possess important distinguishing characteristics. General principles often persist, with refinements and reinterpretations, through multiple scientific revolutions, while formal postulates tend to be specific, limited, and often ultimately inadequate to capture the essence of the principles motivating them. For example, the general principle of covariance, which states that physical laws are observer-independent, motivates the formal postulates of Galilean invariance in Newtonian physics, Lorentz invariance in special relativity, and local Lorentz invariance in general relativity, each new postulate supplanting the last, while covariance itself remains applicable even to nonmanifold models of spacetime. Dubious postulates sometimes persist because of a lack of clear alternatives. For example, the dynamical character of spacetime is widely acknowledged, yet the standard model, as well as some newer theories, fail to incorporate this property. Ancillary assumptions can be particularly troublesome because they tend to escape scrutiny. Historical examples include the luminiferous aether and the assumptions leading to Bell's inequalities.

Six crucial general principles of modern physics are symmetry, conservation laws, covariance, the second law of thermodynamics, background-independence, and causality. These principles are intimately related. Results such as Noether's theorem tie symmetries to conservation laws, and covariance is understood as a symmetry in the context of relativity 3 Alternatively, covariance may be reinterpreted in terms of generalized order theory. Both viewpoints involve separating privileged and unprivileged information, the privileged information being that fixed by a group action or contained in a distinguished suborder. The second law may be understood in a similar context; for instance, entropy is sometimes determined by the cardinality of a Galois group, and entropy in statistical thermodynamics involves the number of microscopic refinements of macroscopic states. Background independence is usually understood as a statement about spacetime, that it is a dynamical entity subject to physical laws such as Einstein's equations, rather than a static object to be taken for granted, such as Minkowski spacetime. More generally, background-independence is an example of the use of parsimony to achieve explanatory and predictive power; the less a theory assumes, the more it can potentially reveal. For example, Einstein's equations in general relativity predict the curvature of spacetime, but not the dimension; a theory whose dynamical laws also predicted the dimension would be superior in an obvious way. Causality is often formalized at the classical level as an irreflexive, acyclic, transitive binary relation on the set of spacetime events. Prediction in physics relies upon the discovery of causal relationships, together with the as- 
sumption of reproducibility. Causality is related to covariance via order theory, to the second law via the arrow of time, and to background-independence via the criteria of explanatory and predictive power. However, the deep meaning of causality, its relation to time, and its appropriate role in quantum theory, are all controversial.

Other general principles deserving mention include symmetry-breaking, superposition, stationary action, the sum-over-histories method, cluster decomposition and other versions of locality, Einstein's equivalence, scale-dependence and independence, the significance of the Planck scale, the holographic principle, dualities such as $S$-duality, and various principles involved in the interpretation of quantum theory. Untested theories rely on further principles whose importance is partially tied to their success. For example, Maldacena's AdS/CFT correspondence [8] becomes central if string theory is assumed valid. Subjects in pure mathematics, such as number theory, also offer general principles and conjectures with deep connections to physics. For example, zeta functions, and hence the Riemann hypothesis, are connected to quantum field theory via noncommutative geometry and the theory of motives [4]. The Langlands program is connected to symmetry and duality in physics through group representation theory and conformal field theory, and thence also to string theory [9].

I now describe the formal postulates of modern physical theories, along with some ancillary assumptions. General relativity postulates a four-dimensional real manifold with Lorentz signature, interpreted as spacetime, whose curvature, interpreted as gravitation, interacts dynamically with matter and energy according to Einstein's equations, and whose metric properties determine the causal order of the universe. Hawking and Ellis [10] showed that singularities arise in the generic case. Several versions of quantum theory exist, of which I will mention two; these versions are equivalent under suitable restrictions. The Hilbert space version postulates complex Hilbert spaces whose elements represent probability amplitudes of point particles, self-adjoint operators whose eigenvalues are interpreted as the possible values of measurements, and time evolution according to the Schrödinger equation. Feynman's sumover-histories version [11] postulates probability amplitudes for point particles given by sums over spacetime paths, with each path contributing equally in magnitude, and with the phase of each path determined by the classical action. This version generalizes easily to different models of spacetime. Quantum field theory postulates the existence of operator fields that create and annihilate states representing point particles in complex Hilbert spaces. States describing particular types of particles correspond to particular representations of symmetry groups. The properties of Minkowski spacetime impose external symmetries described by the Poincaré group. There are also internal symmetries, such as those described by gauge groups. The standard model is constructed from quantum field theory and includes particles, fields, and symmetry groups corresponding to the experimental observations of particle physicists over the last century. 


\section{Unexplained Phenomena}

Since the ascendancy of general relativity and the standard model, a variety of unexplained phenomena have been recognized. The large-scale dynamical anomalies attributed to dark matter and dark energy, the absence of a large cosmological constant arising from vacuum energy, and the apparent asymmetry between matter and antimatter in the observable universe, are a few of the most prominent examples. These phenomena suggest the promise of physical models that naturally incorporate scale-dependence, and that offer statistical or entropic explanations of small nonzero constants and inexact symmetries. Order-theoretic and graph-theoretic models tend to perform well by these criteria.

The dark matter hypothesis is based on the failure of astrophysical systems on the scale of galaxies to obey relativistic dynamics, assuming only the matter content detectable by nongravitational means f $^{4}$ This hypothesis has been compared unfavorably to the luminiferous aether, and various new dynamical laws have been proposed to account for observed behavior without invoking missing mass. However, this phenomenon does behave like ordinary matter in many respects, as observed in the collision of galaxies and in certain examples of gravitational lensing. If the dark matter hypothesis is valid, the matter involved almost certainly falls outside the standard model. Claims have been made of laboratory observations of new particles consistent with dark matter, but these are not broadly accepted at present. Dark energy is the entity invoked to explain the phenomenon interpreted as acceleration of the expansion of the universe. The cosmological constant appearing in the modified form of Einstein's equations is one possible type of dark energy. Predictions based on quantum field theory and the Planck scale yield a value for the cosmological constant roughly 120 orders of magnitude greater than observation implies. Interestingly, causal set theory suggests a fluctuating cosmological constant close to the observed value, based on a simple argument involving discreteness and the size of the Hubble radius in Planck units. Nonconstant models of dark energy, such as quintessence, have also been proposed, but any fluctuations in dark energy appear to occur on much larger scales than those of dark matter or ordinary matter and energy. Anomalies in the motion of certain large galactic clusters, called dark flow, might reflect such fluctuations. Dark matter and dark energy extend the scale-dependence of phenomena already observed in conventional physics. Strong and weak interactions, electromagnetism, ordinary gravity, dark matter, and dark energy all dominate on different scales, each covering roughly equivalent ranges of magnitude. The extent of this scale-dependence was unknown during the development of relativity and quantum theory, and should receive greater emphasis in new models.

Our present understanding of antimatter comes almost entirely from quantum field theory, and it is reasonable to ask if the observed matter-antimatter asymmetry might indicate a problem with quantum field theory itself, or at least with the standard model. Unexpected asymmetries have been successfully handled by quantum field theory in the past; the prototypical example is $\mathrm{CP}$ violation, which is itself related to the matter-antimatter problem. However, potential 
sources of matter-antimatter asymmetry in the standard model seem either too weak, or too strong, to account for observation. Interesting experimental issues regarding antimatter remain to be resolved. For example, proof is lacking that antimatter interacts in the same way as ordinary matter gravitationally, and it has even been suggested that local matterantimatter asymmetry might result from gravitational segregation. More conventionally, a number of experiments designed to investigate matter-antimatter asymmetry have recently produced data suggesting rates for certain decay processes different than those predicted by the standard model. It is too early to render judgment on the significance or meaning of these results, however.

\section{Rejected Assumptions}

The first few assumptions I reject are that spacetime is a manifold, that systems evolve with respect to an independent time parameter, and that the universe has a static background structure. General relativity includes the first assumption, and the standard model includes all three. These assumptions are already widely doubted and are retained largely because of the absence of alternatives. Hence, their rejection is not very revolutionary, but a successful theory abstaining from them would be. Along with these assumptions perish a number of corollaries. Dimension becomes an emergent property, and is no longer assumed to be constant, nondynamical, or an integer. Properties previously viewed as arising from a metric must either be discarded or provided with different justification. For example, given a path between two points in a metric manifold, there exist many other such paths of similar length; however, a nonmanifold model of spacetime might admit a single short path between two events, while every other path is much longer.5 Other such properties could be listed, but more interesting is the question of what mechanisms supply the illusions of these properties at ordinary scales.

The answers I will propose involve reinterpreting the principles of causality and covariance. This requires the rejection of some common assumptions about these specific principles. First, I reject the assumption that the "metric" properties of spacetime involve any information besides the binary relation generating the causal order and an overall scale factor 6 This means, in particular, that spacelike sections are merely unordered sets, with no independent notion of distance or locality. Second, I reject the assumption that the binary relation generating the causal order is transitive. Indeed, information about direct and indirect causation is a priori relevant, and this information is lost by closing the relation under transitivity 7 Third, I reject the assumption that this relation is acyclic 8 Causal cycles, if they exist, should be viewed as properties of a binary relation, not as self-erasing paths on a background structure. Turning to covariance, I reject the assumption that covariance is an instance of group symmetry; rather, covariance should be viewed in order-theoretic terms 9 This is notable because progress in physics has historically derived from invoking new symmetry principles, rather 
than rejecting existing ones. Group representation theory lies at the heart of modern physics as the mathematical expression of symmetry, and remains perhaps the most likely vehicle for initial, verifiable progress beyond the standard model; however, analogous constructs from order theory, and perhaps other notions more primitive than groups, will likely partially replace representation theory over the long term. Finally, I reject the assumption that spacetime is commutative. A number of existing proposals about spacetime microstructure lead naturally to noncommutative spaces in the sense of Connes [3] via the deformation theory of Hopf algebras 10 but noncommutative geometry is relevant more generally, and even classical spaces such as Minkowski spacetime possess important noncommutative structures.

\section{New Principles}

The central new principle I propose is the causal metric hypothesis, which states that the metric properties of classical spacetime, up to overall scale, arise from a binary relation, which I will call a causal relation, on a set, which I will call a universe, and that the phase associated with a congruence class of directed paths in the configuration space of such universes is determined by the causal relations of its constituent universes. Mathematical tools necessary to implement these ideas include a synthesis of multicategory theory and categorification in abstract algebra, involving interchangeability of objects, morphisms, elements, and relations; a version of graph 11 dynamics more sophisticated than versions involving uniform probabilities; and the theory of noncommutative algebras over sets with partially defined operations. In particular, path algebras encode the properties of both individual universes and subgraphs of their configuration spaces, while providing convenient methods of computation.

A causal relation may be viewed as a generalized partial order. The most interesting cases I know of involve one or more additional assumptions such as acyclicity, local finiteness, interval finiteness, and various predecessor and successor conditions ${ }^{12}$ Countability is a natural assumption in the locally finite or interval finite case, but this is only an assumption about cardinality, not order; in particular, countable acyclic universes are generally far too complicated to embed ${ }^{13}$ into the natural numbers or integers ${ }^{14}$ Local properties are generally more reasonable to impose than nonlocal properties because of our ignorance of the global structure of the universe ${ }^{15}$ Recovery of a Lorentzian manifold from a physically relevant causal relation is necessary at some level of approximation because of the success of relativity, at least on appropriate scales. Metric recovery theorems for Lorentzian manifolds show that volume as well as order data is necessary to fully specify the geometry. The simplest way to specify volume data is by means of a constant discrete measure, which is the approach of causal set theory. Such a measure contains no independent information besides an overall scale factor, and thus satisfies the causal metric hypothesis. However, the causal metric hypothesis also allows for other methods of specifying volume data that depend on the causal relation in more complicated ways. 
Turning to quantum theory, the sum-over-histories method applies naturally in the acyclic case. Sums involve paths in a configuration space of universes, which I will call a causal configuration space ${ }^{16}$ This space inherits a transitive binary relation induced by special morphisms among its universes, which I will call transitions ${ }^{17}$ Transitions replace the notion of time evolution; the target of a transition may be viewed as a later stage of development of its source. Hence, universes are viewed as elements and transitions as relations in the higher-level structure of causal configuration space. I will call the relation induced by these transitions the universal relation, and each of its subrelations a kinematic scheme. Under suitable assumptions, a pleasing fractal picture emerges, in which kinematic schemes share most of their abstract properties with the causal relations of their constituent universes, and quantization becomes an iteration of structure, at least locally 18 A kinematic scheme specifies families of directed paths terminating at each of its universes, which may be viewed as possible interpretations of their histories 19 The sum-over-histories method assigns an amplitude ${ }^{20}$ to each bipartite family of universes in a kinematic scheme ${ }^{21}$ This amplitude is interpreted as encoding the probability that a universe in the initial family will evolve into a universe in the terminal family. It is computed by summing quantities called phases over congruence classes of paths between the two families ${ }^{22}$ Just as it is useful to view universes as elements and morphisms as relations, it sometimes helps to view relations, and hence morphisms, as elements ${ }^{23}$ Technical advantages result from working exclusively with relations in a kinematic scheme. The set of relations inherits an induced binary relation from the universal relation. This viewpoint, together with the sum-over-histories method, enables the derivation of a dynamical equation which I will call the universal Schrödinger equation 24

Recovery of established physics at appropriate scales from these principles is a challenging problem due to their parsimony, with the standard model likely more difficult to recover than general relativity. It is natural enough to imagine classical spacetime emerging from causal relations; discrete causal theories such as causal set theory and causal dynamical triangulations have not yet achieved this goal, but these theories have not yet received the attention and effort lavished on string theory and loop quantum gravity. Moreover, they involve assumptions such as transitivity, constant discrete measure, interval finiteness, isomorphism with a suborder of the integers, and particular versions of covariance, all of which are open to criticism. Emergence of Minkowski spacetime is the first step toward the standard model as well as toward relativity, since along with it will emerge Hilbert space and the Poincaré group together with its representation theory, which enable the most fundamental considerations of particle states in quantum field theory. However, particle states are added as separate ingredients to Minkowski spacetime in quantum field theory, while in the present context both must emerge together 25 Based on our best guesses about the fundamental scale, the simplest of "elementary particle" interactions might easily involve Avogadro's number of fundamental elements, or its square, or its cube. This is encouraging in the sense that such magnitudes allow for mechanisms such as entropy and graph dynamical-phase transitions to produce sharp behavior and select for precise quantities; it is discouraging in the sense that interactions large enough to observe might be difficult to model. 


\section{Practical Considerations}

The empirical status of the rejected assumptions and new principles discussed here is mixed. Manifold structure is still experimentally tenable, but an independent time parameter and static background structure have been in serious doubt ever since the first observations supporting general relativity. It is impossible to disprove these notions, but one may reasonably hope to do without them. Conversely, it is impossible to prove the causal metric hypothesis, but it could be disproven by evidence of multiple independent relations on the power sets of universes ${ }^{26}$ This would require generalization of the methods introduced here. Intransitivity of the binary relation generating the causal order is self-evident at large scales. At the fundamental scale, the question is whether or not intransitive relations should be included in the sum-over-histories; the two methods may or may not predict distinct physics. I know of no solid evidence for causal cycles, but the history of physics includes a number of interesting interpretations of existing phenomena that include them. Radical noncommutativity of spacetime and the inadequacy of the symmetry interpretation of covariance might be demonstrated only in conjunction with breakdown of manifold structure; milder noncommutative notions apply to existing models, and are already entering the mainstream.

At the time of this writing, the Large Hadron Collider (LHC) at CERN has reported the detection of a new particle with energy near $125 \mathrm{GeV}$ and properties similar to the predicted properties of the standard model Higgs boson. Further work is necessary to analyze possible deviations from these predictions, but there is concern in the experimental physics community that the observed particle will precisely match the standard model Higgs, that the LHC will fail to detect any other new physics, and that an experimental dark age will ensue. New fundamental physics may soon be directly observable only at technologically or economically unattainable energies. Even in the distant future, it is difficult to imagine directly probing energies approaching the Planck scale. These considerations highlight the need for creative ideas about what experimental phenomena to search for and how to search for them.

An intriguing possibility is that fundamental-scale physics might be simulated at larger scales by means of quantum circuits ${ }^{27}$ Such circuits are small at ordinary scales, but are many orders of magnitude larger than the Planck scale, and while only very simple quantum circuits presently exist, complex circuits are not far off. If spacetime has a sufficiently simple structure, appropriate quantum circuits might model certain interactions isomorphically, creating virtual fundamental-scale laboratories easily accessible to existing technology. Such simulations might imply unforeseen phenomena that could then be detected independently at reasonable scales. Meanwhile, results one might look for in traditional experiments include inexactness of symmetries, variation or small nonzero values of physical constants, and new kinds of scale dependence. Finally, the dimension of space as well as its curvature might vary with "energy density," though the effect might be immeasurably small. 


\section{Notes}

11 Order has a more general meaning here than that of a reflexive, antisymmetric, transitive binary relation, which is the usual definition of a partial order. Here an order is simply the transitive closure of a binary relation, which is said to generate the order. The transitive closure is the minimal transitive binary relation containing the original binary relation.

2 The classical version of the causal metric hypothesis is analogous to Rafael Sorkin's maxim [7 that "order plus number equals geometry," but is more general and involves a different meaning of order.

3 Local symmetry, of course, in general relativity.

4 In contrast, objects near the stellar scale seem to verify relativistic predictions remarkably well.

5 This requires, of course, an alternative notion of distance. The result is an altered notion of locality.

6 This factor is necessary because a binary relation carries no canonical measure-theoretic information.

7 The usual transitive notion of causality may be recovered by taking the transitive closure.

8 This involves no paradoxes of the sort arising from clashes between distinct metric and causal structures.

9 For example, different frames of reference in relativity may assign different time orders to events separated by spacelike intervals; these orders represent different classes of refinements of the causal order.

10 The class of theories called deformed special relativity (DSR) falls into this category. Whatever their shortcomings, these theories draw attention to important and unresolved issues.

11 Directed graphs and binary relations are interchangeable in this context. For instance, the continuum corresponds to a locally uncountable directed graph with a vertex for each real number and a directed edge for each relation; hence the set of relations corresponds to the open half-plane above the diagonal. Locally countable and particularly locally finite graphs are probably of greater physical interest, however.

12 Local finiteness means that each element has a finite number of direct predecessors and successors, where $x$ is said to precede $y$ and $y$ to succeed $x$ if $x<y$ in the transitive closure of the causal relation. Interval finiteness means that the number of elements succeeding a given element and preceding another is finite. An example of a predecessor condition is that the number of maximal predecessors of each element is finite. Note that this is possible even if the number of direct predecessors is infinite.

13 An embedding is an injective morphism, and a morphism is a map preserving the causal relations.

14 For example, consider Jacob's ladder, the universe with elements $\left\{x_{0}, x_{1}, \ldots\right\} \cup\left\{\ldots, y_{-1}, y_{0}\right\}$ and relations $x_{i}<x_{i+1}, x_{i}<y_{-i}$, and $y_{j}<y_{j+1}$. It is acyclic, locally finite, and countable, but does not embed into any well-ordered set, or the integers.

15] In particular, local finiteness is a better condition in this sense than interval finiteness.

16 For example, the causal configuration space of causal set theory is the space of all acyclic, transitive, interval finite universes admitting an embedding into the natural numbers. 
17 A transition is an embedding of one universe into another such that the complement and the past of the image are disjoint. A composition of transitions is a transition, so the relation is transitive. There is no loss of information associated with transitivity in this case, because all the information is inherited from the constituent universes. However, irreducible transitions are important when they exist.

18 This is an example of the algebraic tool known as categorification, in which structure is added or ignored by promoting elements or demoting objects. Note the similarities, and differences, to the naïve process of quantizing by promoting variables to operators. Also compare this to the theory of moduli spaces in algebraic geometry, where each point on a special algebraic scheme is itself an algebraic scheme, and to other analogous situations enjoying enough rigidity to control the sizes of configuration spaces.

19 For example, the kinematic scheme of sequential growth dynamics in causal set theory is the subgraph of causal configuration space given by irreducible transitions, which in this case are transitions adding a single element to their initial universes. Alternatively, kinematic schemes involving transitions that add entire generations to their initial universes govern discrete-time analogues of relativistic dynamics.

20 In ordinary quantum theory, probability amplitudes are complex-valued. In the present context, amplitudes arise from the causal relations, and the complex numbers cannot be taken for granted. Finite algebraic structures provide interesting alternatives.

21 A bipartite family is a pair of families such that each universe in the initial family is related to at least one universe in the terminal family via a transition, but no pair of universes in either family is so related.

22 A partial history is represented by a congruence class of paths in a kinematic scheme, where two paths are congruent if and only if they share the same initial and terminal universes. Alternatively, one may sum over individual paths; in this case, there is surplus information and hence a covariance principle.

23 This is an example of the algebraic notion of a multicategory, in which the morphisms of one category are the objects of a higher category.

24 This method originates with Feynman [11, who thereby re-derived the usual Schrödinger equation for nonrelativistic quantum theory. A special case of such an equation is

$$
\Psi_{R}(r)=\theta(r) \sum_{r^{\prime}<r} \Psi_{R}\left(r^{\prime}\right)
$$

where $R$ is a subset of the relation set of a kinematic scheme, $r, r^{\prime} \in R$ are relations, $<$ is the induced relation on $R$, and $\Psi$ is the past causal wave function defined by $\Psi_{R}(r):=\sum_{s \in S_{M}(R ; r)} \theta(s)$, where $S_{M}(R ; r)$ is the set of maximal irreducible directed paths in $R$ terminating at $r$, and $\theta$ is the phase map.

25 Of course, one could attempt to introduce matter and energy as auxiliary data, but this would defeat the purpose of the project; since spacetime interacts causally with matter and energy, the causal relation must subsume all three to be worthy of its name.

26 The simplest case, involving two independent binary relations, is the causal analogue of two classical time dimensions. This seems quite radical to me, but others have attempted to replace linear time with Bloch spheres, etc.

27 Such circuits may be represented by acyclic directed graphs whose edges are weighted by single-qubit unitary transformations. Abstractly, these graphs are causal universes with causal relations whose refinements are generalized frames of reference. Conversely, many such universes correspond to quantum circuits, although there are important restrictions, arising for instance from the no-cloning theorem. 


\section{References}

[1] Katrin Becker, Melanie Becker, and John Schwarz. String Theory and M-Theory: A Modern Introduction. Cambridge University Press, 2007.

[2] Thomas Theimann. Modern Canonical Quantum General Relativity. Cambridge Monographs on Mathematical Physics. Cambridge University Press, 2007.

[3] Alain Connes. Noncommutative Geometry. Published online, 1990. English version of Géométrie non commutative, published by InterEditions Paris.

[4] Alain Connes and Matilde Marcolli. Noncommutative Geometry, Quantum Fields and Motives. Published online, 2007.

[5] Erik Verlinde. On the Origin of Gravity and the Laws of Newton. Journal of High Energy Physics, 2011, Volume 2011, Number 4, 29.

[6] D. P. Rideout and R. D. Sorkin. A Classical Sequential Growth Dynamics for Causal Sets. Physical Review D, 61(024002 (2000)), 2004.

[7] Rafael Sorkin. Light, Links and Causal Sets. Journal of Physics Conf. Ser. 174: 012018, 2009.

[8] Juan Maldacena. The Large $N$ Limit of Superconformal Field Theories and Supergravity. International Journal of Theoretical Physics, 38(4):1113-1133, 1999.

[9] Edward Frenkel. Lectures on the Langlands Program and Conformal Field Theory. Published online, 2005. Based on lectures given by the author at the DARPA workshop "Langlands Program and Physics" at the Institute for Advanced Study, March 2004.

[10] S. W. Hawking and G. F. R. Ellis. The large-scale structure of space-time. Cambridge Monographs on Mathematical Physics. Cambridge University Press, 1973.

[11] R.P. Feynman. Space-Time Approach to Non-Relativistic Quantum Mechanics. Rev. of Mod. Phys., 20, 367, 1948. 